\title{
Une méthode de recherche et de formation :
}

La construction d'un organigramme modulable du fonctionnement de la classe en biologie. Enseignements, prolongements, spécificité

\section{Christiane Haguenauer et Jean-Paul Doste}

\section{(2) OpenEdition}

1 Journals

Édition électronique

URL : http://journals.openedition.org/trema/2078

DOI : $10.4000 /$ trema.2078

ISSN : 2107-0997

Éditeur

Faculté d'Éducation de l'université de Montpellier

\section{Édition imprimée}

Date de publication : 1 septembre 1996

Pagination : 155-167

ISSN : 1167-315X

\section{Référence électronique}

Christiane Haguenauer et Jean-Paul Doste, « Une méthode de recherche et de formation : », Tréma [En ligne], 9-10 | 1996, mis en ligne le 18 septembre 2013, consulté le 01 mai 2019. URL : http:// journals.openedition.org/trema/2078; DOI : 10.4000/trema.2078

Ce document a été généré automatiquement le 1 mai 2019.

Trema 


\section{Une méthode de recherche et de formation:}

La construction d'un organigramme modulable du fonctionnement de la classe en biologie. Enseignements, prolongements, spécificité

\section{Christiane Haguenauer et Jean-Paul Doste}

\section{Introduction}

1 Une pratique de formation des enseignants du premier et du second degré consiste à mettre les stagiaires en situation d'observateurs du fonctionnement de la classe. Mais le nouveau professeur est souvent submergé par la complexité et la variété des faits, des actions, des décisions; pour faciliter sa tâche, il est d'usage de lui fournir une grille d'analyse. En fait, ce type d'outil est utile surtout pour ceux qui ont participé à sa construction, qui ont choisi et clarifié les paramètres jugés essentiels de l'acte d'enseignement observé. La participation à une telle expérience, dans un groupe de recherche interdisciplinaire animé par J. Berbaum dans le cadre de l'IREM pendant la décennie 70-80, a prouvé son efficacité. Face à ce constat, nous avons élaboré un outil qui sert de médiateur entre les stagiaires et les formateurs, en favorisant le dialogue pour susciter questionnement, clarification et modifications en retour tout en identifiant, dans cette construction progressive, les variables spécifiques de la biologie. Cet organigramme a été testé comme instrument de communication entre les participants à un atelier des Rencontres Européennes de Didactique de la Biologie, pour rechercher ensemble, ce qui, dans l'acte d'enseigner, caractérise la biologie. Nous allons résumer son élaboration progressive en formation avant de présenter l'apport des différents acteurs de l'atelier pour ensuite s'interroger sur les prolongements de la démarche d'utilisation de l'outil. 


\section{L'organigramme, outil de dialogue et de construction de grilles modulables appropriées}

\section{1. Élaboration de l'outil en formation des enseignants}

2 L'idée de l'outil est venue à la suite de la contribution conjointe, par les auteurs, à l'animation de deux universités d'automne organisées par l'IUFM de Lorraine et la MAFPEN de Nancy-Metz, avec la collaboration de Professeurs de l'Université. Les thèmes en étaient respectivement «Rôle et place de la pratique clans la formation initiale et continue des enseignants » et "Polyvalence des Professeurs d'école»; ils mettaient en relief la nécessité de critères clairs pour analyser et dépasser les situations concrètes et rendre efficiente l'interaction entre théorie et pratique. Mais les échanges entre participants ont prouvé que, le plus souvent, tout en disposant d'un arsenal de grilles d'observation de situations pédagogiques, les formateurs ne les utilisaient pas, ou pas durablement.

\subsubsection{Observation de l'action des Professeurs spécialistes des Sciences de la Vie et de la Terre}

3 Il est de tradition de proposer aux Professeurs de SVT formateurs et stagiaires dans les lycées et collèges, des grilles d'analyse du fonctionnement de la classe, même si aucune ne s'affiche comme spécifique de l'enseignement de la Biologie. Il est aussi habituel de demander aux uns et aux autres, au cours de l'année, des rapports d'évaluation, formative puis sommative, qui prouvent que chacun traduit sa réflexion dans un langage qui lui est propre, ce qui rend l'expérience difficile à transmettre. Nous voulons pourtant que chacun dispose d'un outil modulable, utilisable dans une perspective formative d'appréciation de la pratique, favorable aux échanges et à la distanciation. C'est pourquoi, pour mobiliser tuteurs et stagiaires, nous lançons le dialogue sur un organigramme ouvert, qui trouve ses racines dans la formation.

Dans un premier temps, nous avons proposé les grilles classiques aux différents tuteurs, afin que chacun détermine, en réunion de conseillers puis avec ses stagiaires, les variables qu'il lui semblait indispensable de contrôler dans les situations pédagogiques vécues; chaque groupe formé par un tuteur et son stagiaire a été incité à construire sa propre grille, modulée avec le temps. La confrontation des différentes grilles produites contribuait à en montrer les insuffisances : ambiguïté des descripteurs, absence de prise en compte de l'évolution de l'action et des interrelations entre les différents items qui donnent cohésion et cohérence à l'outil utilisé, manque d'éléments d'appréciation globale (sommation des critères, échelle de hiérarchie...) pour effectuer l'évaluation finale. Au delà des difficultés d'utilisation de ce type d'outil, la collaboration a fait apparaitre des permanences et la nécessité de valoriser l'essentiel en catégorisant les actions, en favorisant les interrelations entre variables tout en permettant les échanges entre les stagiaires et formateurs; d'où la construction de l'organigramme pour relier les rubriques correspondant à ce que nous retenons de commun aux différentes grilles et jugeons essentiel dans l'appréciation de la performance du stagiaire. Ces rubriques sont regroupées en deux ou trois catégories qui portent sur la préparation scientifique et 
didactique et sur la mise en œuvre pédagogique ; c'est au travers des interdépendances entre ces deux ou trois approches que s'expriment les participants au dialogue (fig. 1).

\subsubsection{Observation de l'action des enseignants polyvalents : les professeurs d'école}

5 La construction, avec des professeurs d'école, d'une grille d'observation du fonctionnement de la classe impose que cet outil leur permette d'analyser des situations pédagogiques non spécifiques à une discipline. En effet, la formation de ces enseignants polyvalents vise la polydisciplinarité (maîtrise des contenus des différentes disciplines enseignées à l'école primaire) et la polycompétence (capacité à préparer, conduire, analyser différentes situations pédagogiques, à évaluer les performances d'élèves, à remédier aux difficultés). Ainsi, même s'il s'appuie sur des situations d'enseignement en biologie, l'outil doit permettre d'analyser d'autres situations en français, en mathématiques, en histoire... D'où notre démarche pour favoriser la production d'un organigramme successivement spécifique et pluridisciplinaire, suffisamment précis pour être utilisé en biologie mais extensible à l'ensemble des autres disciplines enseignées. La procédure utilisée se déroule progressivement.

Figure 1 : Organigramme de synthèse provisoire élaboré à l'IUFM de Lorraine.

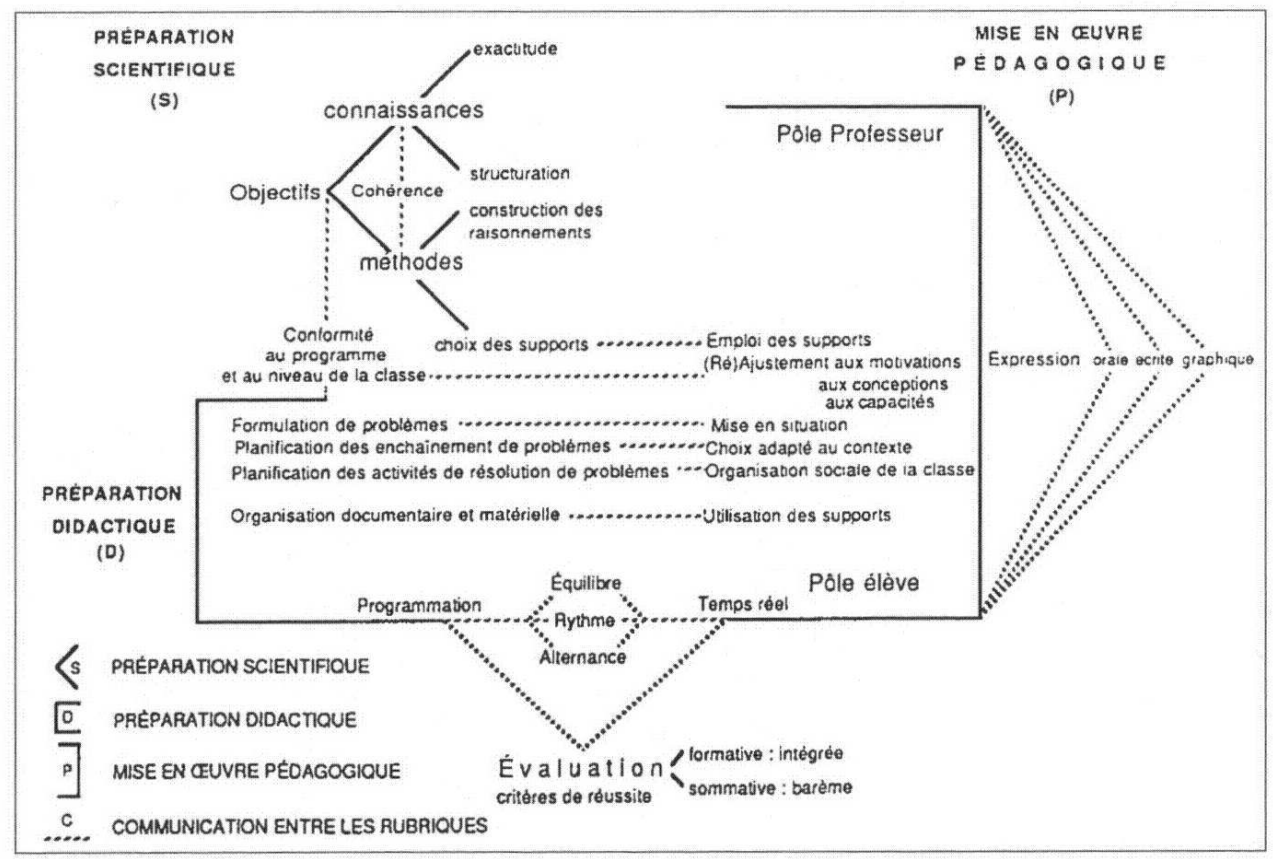


Figure 2 : Organigramme de travail soumis aux participants à l'atelier.

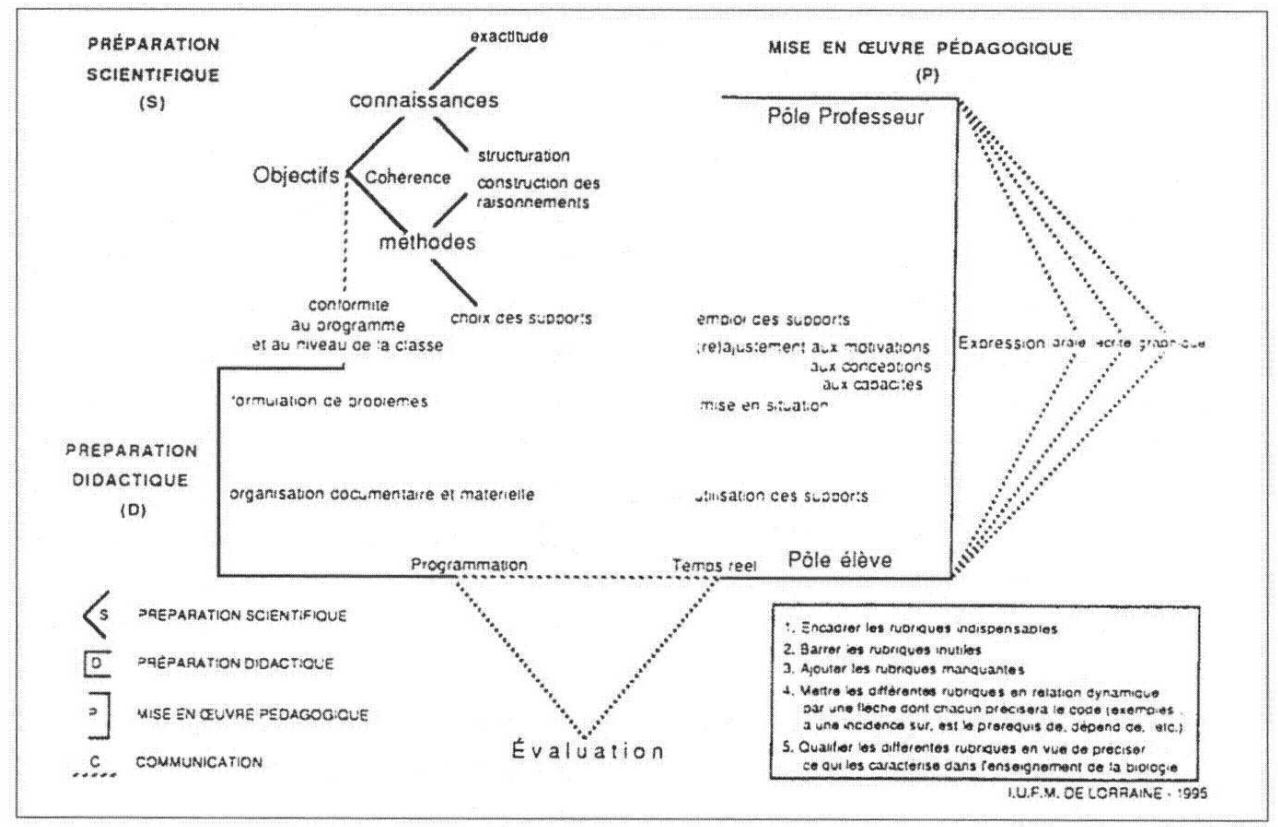

6 Un premier repérage des variables de l'action pédagogique est assuré par l'utilisation d'un document vidéo où des enseignants jouent à mettre en œuvre un style d'enseignement différent sur un même objet d'enseignement puis à construire un outil d'observation des interactions enseignants/savoir/élèves sous la forme de questions essentielles.

7 Les stagiaires utilisent ensuite un questionnaire clans une situation d'observation de tuteur et/ou de pair en biologie et dans des disciplines diverses ; il s'ensuit des remarques sur la difficulté liée à l'observation et à l'application d'un tel instrument (vocabulaire trop didactique, trop spécialisé, sans interrelations entre les questions, sans repérage de l'action dans le temps); elles appellent de difficiles modifications individuelles de l'outil, liées aux contenus, au contexte, aux dispositifs et aux matériels différents suivant les situations concernées. La suggestion de construire une grille prenant en compte l'ensemble des remarques a conduit formateurs et stagiaires à élaborer un organigramme.

\subsubsection{Les nécessaires clarifications}

8 Munis de l'organigramme, les stagiaires, professeurs de lycées et collèges ou d'école, vont tester, sur le lieu d'exercice de leur pratique avec les tuteurs ou formateurs, l'efficacité de cet outil qui devient un instrument de dialogue et de clarification conceptuelle :

- instrument de dialogue, il permet de préciser, d'expliciter les critères d'observation choisis et donc de désigner des descripteurs d'action, de rendre compte des différents sous-jacents théoriques des uns et des autres, de préciser les concepts des différentes didactiques, en particulier ceux de la biologie.

- instrument de clarification conceptuelle, il donne l'occasion de s'expliquer, au niveau requis, sur les termes utilisés dans les différentes rubriques et sur leur signification. Il s'ensuit une succession d'écrits collectifs, aidés du formateur, pour les différentes rubriques de chacune des catégories : aboutissements provisoires, ils expriment la dynamique de 
construction de l'outil, enrichi ou reconsidéré sous l'éclairage des expériences et/ou acquis théoriques nouveaux.

Ce travail comportant une interrogation sur les variables spécifiques de la biologie, nous avons voulu connaître l'avis des participants à l'atelier correspondant aux Rencontres de Montpellier.

\subsection{Apport de l'outil et spécificités de la Biologie pour les participants à l'atelier}

10 L'atelier a réuni dix biologistes: Mondher ABROUGUI, Maria ARCA, Guillemette BERTHOU, Sylvie CAENS MARTIN, Daniel faVRe, Claudine LEBorGNE-TAHIRI, Alexandra MENDES GUERRA FERREIRA, Valdiodio NDIAYE, Daniel ROUSSELET et Guy RUMELHARD ; ces participants, aux fonctions diverses dans le domaine de la didactique, se sont organisés en cinq groupes.

11 En présentant l'historique et la problématique de notre travail puis les buts poursuivis, nous avons soumis aux groupes l'organigramme précédent, partiellement évidé pour amorcer le dialogue sans nuire à l'expression individuelle des priorités (fig. 2).

12 Les membres de l'atelier ont alors disposé d'un temps de réflexion pour échanger leurs points de vue dans chaque groupe et exprimer, par écrit sur transparent, leurs conceptions sur les rubriques et sur leur interdépendance avec d'autres rubriques de leur choix. Aidés de l'outil ainsi réorganisé, ils ont fait part au grand groupe de leurs différentes observations.

13 Notons que les participants pouvaient se procurer, au centre de l'atelier, des définitions de concepts pouvant appartenir à l'organigramme et formulées à différents niveaux en formation à l'IUFM.

\subsubsection{Groupe 1}

Pour ce groupe, formé d'un professeur de l'enseignement secondaire portugais et d'un formateur de l'enseignement agricole français, l'ébauche d'organigramme vaut par la dynamique de construction qu'elle engage et par le contrôle du projet par l'action réalisée dans la phase de mise en œuvre. Les manques à combler portent sur l'observation des élèves. Reprenant des termes de l'organigramme (connaissances, méthodes, cohérence, formulation de problèmes) le groupe les relie entre eux en notant que c'est le niveau de formulation des concepts à s'approprier qui justifie la cohérence entre connaissances et méthodes. L'organigramme complété vise à établir la relation entre le choix initial des supports lié à une organisation documentaire préalable, d'une part, et l'emploi de ces supports dans la réalité de la classe d'autre part : il met ainsi l'accent sur la notion de réajustements de l'emploi des supports avec une référence au vivant qui constitue la spécificité de l'enseignement de la biologie (fig. 3). 
Figure 3. Groupe 1. Organigramme construit par les membres du groupe 1 de l'atelier.

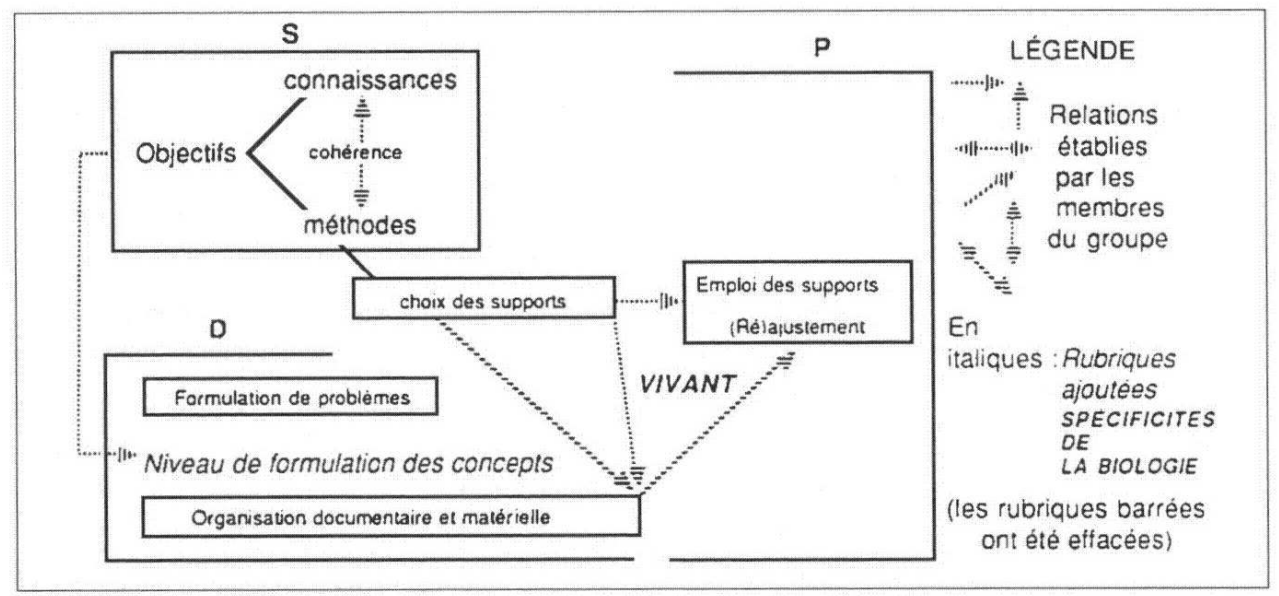

\subsubsection{Groupe 2}

Constitué de deux praticiens et formateurs universitaires à la fois, en France et au Sénégal, ce groupe s'est attaché dans un premier temps à éluder la polémique possible de la distinction entre didactique et pédagogie, en simplifiant l'outil par le regroupement des éléments de l'organigramme en deux ensembles : avant la classe d'une part, pendant et après la classe d'autre part. Avant la classe, le groupe propose de remplacer préparation didactique par une expression plus simple: "comment s'y prendre » ou « organisation de ce qui va se passer. » Le souci de ne pas fournir un outil aux rubriques trop diversifiées tient au fait que certains enseignants ont du mal à exprimer ce qu'ils font, bien qu'ils soient efficaces dans leur action.

L'interrogation du groupe a porté sur l'exactitude des connaissances et sur les méthodes. L'exactitude est factuelle et conceptuelle: factuelle, elle est remise en cause par les niveaux de formulation des concepts qui constituent le domaine de validité de ces connaissances ; sur les concepts il n'existe pas de définition de l'exactitude.

Dans une perspective biologique, le terme de méthode est trop vague, trop général. Il faudrait, disent ces formateurs, particulièrement en biologie,préciser que la mise en œuvre de raisonnements scientifiques appelle validation et réfutation, essentielles pour justifier l'idée d'un savoir non stabilisé.

18 Le groupe réfute l'emploi du mot communication, pour son manque de clarté, lié à un usage abusif, et propose une expression plus pertinente du type: «relation entre les rubriques considérées (fig. 4). 
Figure 4. Groupe 2. Organigramme construit par les membres du groupe 2 de l'atelier.

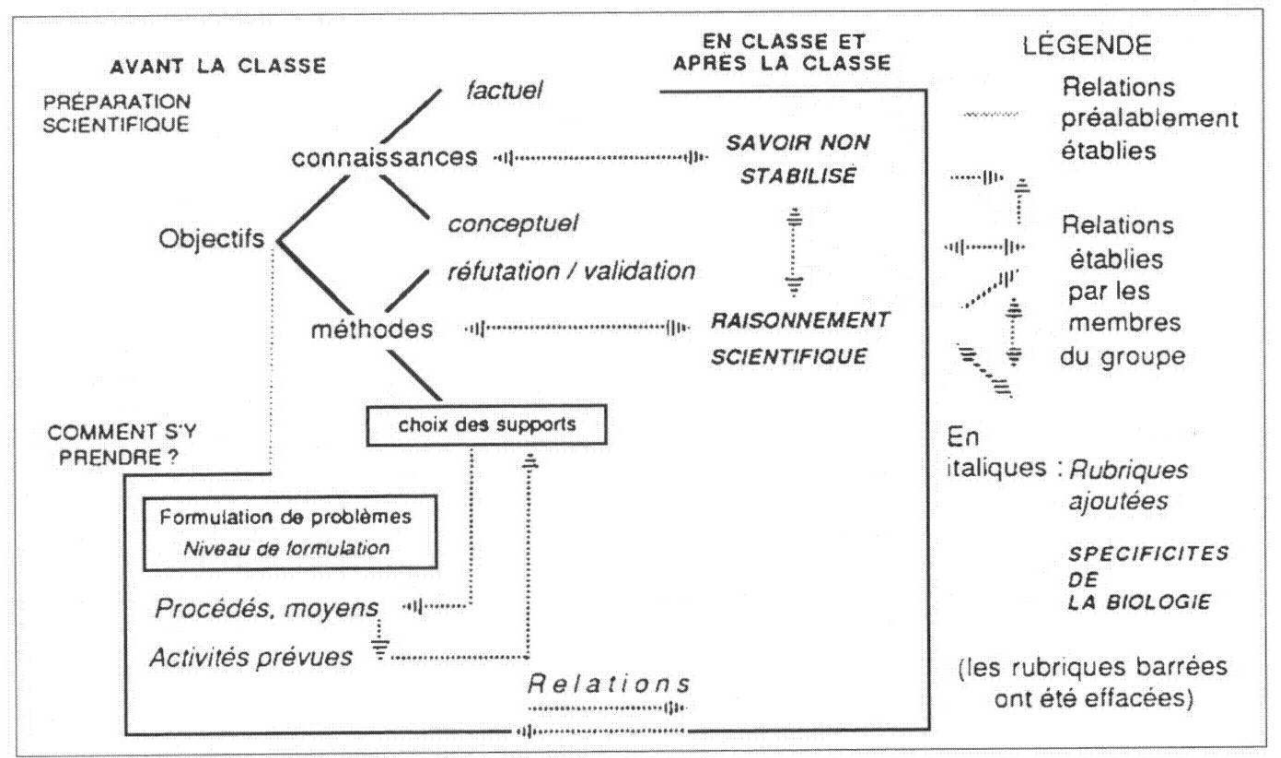

\subsubsection{Groupe 3}

19 Ce groupe, qui comprend un inspecteur-formateur de l'Éducation belge et un praticien et formateur universitaire français, hésite avant de s'approprier l'organigramme. Globalement, l'outil est axé sur le travail du professeur qui intervient dans les trois catégories de rubriques; il importe d'y mettre en bonne place l'élève, pour son rôle régulateur dans la catégorie pratique pédagogique (fig. 5).

Les éléments ajoutés par le groupe concernent l'évaluation, avec en particulier les critères de réussite; ils portent aussi sur l'institution, soulignant et précisant en particulier, dans la phase préparatoire, le rôle de l'institution et de la société. Ce groupe enrichit l'ébauche d'organigramme de la représentation des rétroactions, " permanentes dans l'acte pédagogique. »

21 Une proposition est avancée, pour la formation de professeurs d'école, consistant à faire construire cet outil sous la forme d'un jeu où les acteurs auraient des cartes de couleurs différentes selon les pôles du triplet didactique ; ils devraient compléter progressivement ces cartes et les agencerentre elles (hiérarchisation, inclusion, dichotomie...) en recherchant des critères et en s'aidant d'informations fournies par les formateurs. Difficultés, hésitations, agencements aberrants seraient ainsi l'occasion de clarifications conceptuelles. 
Figure 5. Groupe 3. Organigramme construit par les membres du groupe 3 de l'atelier.

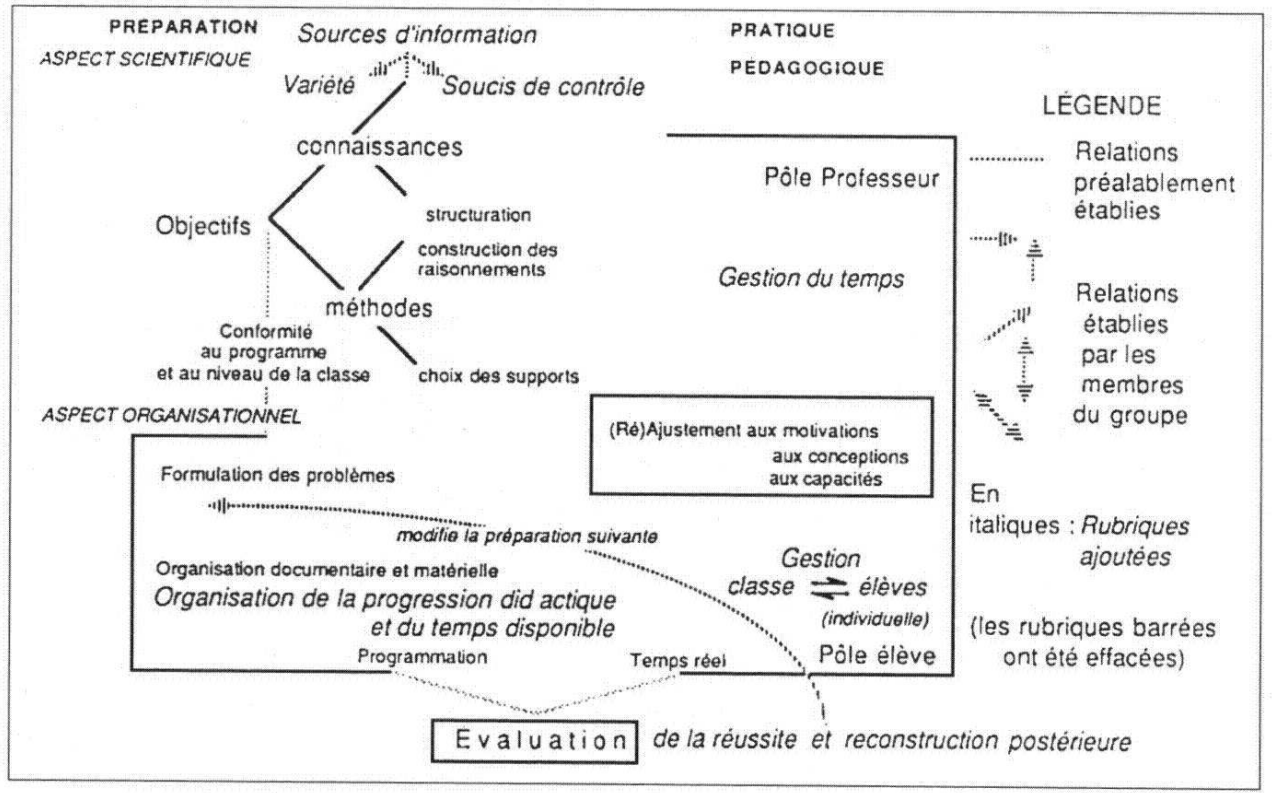

\subsubsection{Groupe 4}

Ce groupe formé de deux chercheurs universitaires, en Italie et en France, a centré sa recherche sur l'explicitation et la mise en relation dynamique de quatre rubriques: connaissance, formulation de problèmes, (ré)ajustement et expression. Partant du programme et du contenu à enseigner, il relie la formulation de problèmes au sens donné dans le contexte de la classe et termine par l'expression.

Essentielle, la rubrique connaissance appelle à délimiter un champ de validité qui oriente la structuration des connaissances et la formulation des problèmes en tenant compte des programmes et du niveau de la classe. Le groupe insiste sur l'étape importante de formulation des problèmes : c'est surtout au travers de sa planification et des techniques disponibles (emploi des documents et supports matériels...) que le savoir apparaît aux élèves (fig. 6).

Du degré de pertinence de cette formulation et de la mise en situation qu'elle constitue dépend l'entretien des motivations et l'expression des conceptions et capacités des élèves et des enseignants.

A mi-chemin des pôles Professeur et Élève, l'accent est mis sur le « ré » de réajustement car, dit le porte-parole du groupe, quelle que soit l'organisation préalable des contenus et des problèmes, leur résolution dépend de la situation de classe. Un ensemble de réajustements crée, dans la classe, la dynamique qui développe la créativité et l'autonomie de l'élève. Pour cela, poursuit le chercheur, le professeur doit éviter d'évaluer et favoriser les phases de validation par les élèves.

Le groupe valorise l'articulation des trois rubriques précédentes par l'expression orale, écrite et graphique qui sert de support à l'évaluation (fig. 6). 
Figure 6. Groupe 4. Organigramme construit par les membres du groupe 4 de l'atelier.

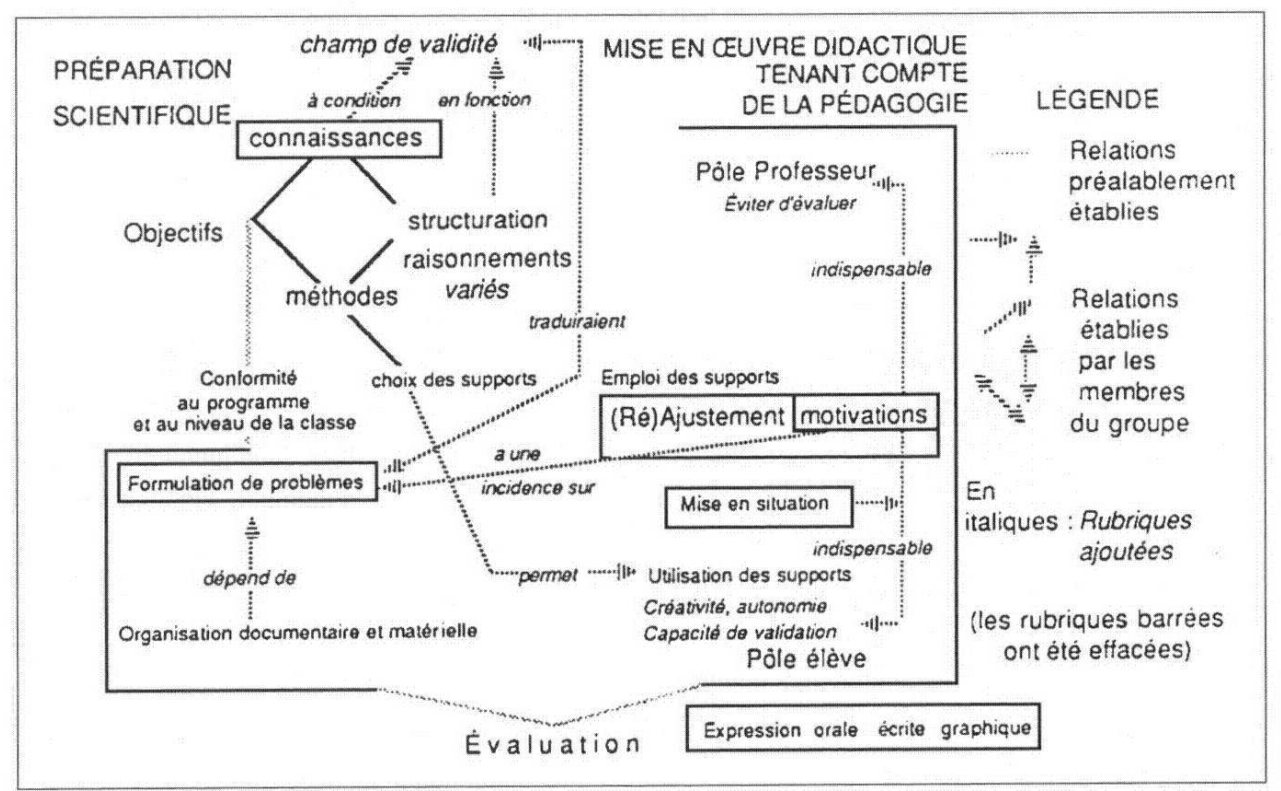

\subsubsection{Groupe 5}

Deux enseignants-chercheurs universitaires français ont participé à une partie seulement des activités. La personne du groupe qui clôt l'atelier était arrivée en fin de phase de recherche préalable à la mise en commun : elle a donc reçu l'organigramme de synthèse élaboré à l'IUFM et destiné, après l'atelier proprement dit, à être confronté aux priorités du groupe dans une nouvelle synthèse; cette condition particulière nous a donné l'opportunité de lui proposer de le réajuster, sous l'éclairage de ses propres convictions et du vécu de l'atelier.

Une suggestion porte sur le remplacement de communication, mal venu pour introduire la conformité au programme imposé par les textes; une autre suggestion consiste à mettre en bonne place l'évaluation de l'enseignant par l'institution et la société, à la jonction de la préparation et de la mise en œuvre, ce qui fait re-situer l'évaluation par l'enseignant dans le cadre de l'organigramme; de même que la mise en œuvre pédagogique fait reconsidérer les objectifs, l'élève modifie en retour l'action du professeur ce qui fait valoriser le feed-back dans les relations (fig. 7).

\subsection{Enseignements de l'atelier et perspectives d'utilisation de l'organigramme}

29 Le travail de construction de l'organigramme, avec stagiaires et tuteurs, puis de (re)construction d'un tel outil en atelier montre que cette construction, génératrice de dialogue et de clarification, a permis de s'interroger sur la spécificité du fonctionnement de la classe en biologie. 


\subsubsection{Enseignements de l'atelier}

En ce qui concerne le premier point, les propositions échangées par les membres de l'atelier font écho aux clarifications conceptuelles sollicitées en formation, même si, pris par la situation proposée, les membres de l'atelier, experts, n'ont pas consulté les rubriques mises à leur disposition. Le souhait de simplifier les outils les a conduit à dialoguer, repérer les manques, noter les relations, expliciter les termes. La (re)construction de l'outil développe l'idée d'interactions, d'interrelations des différentes rubriques et montre la nécessité d'une approche systémique de l'action pédagogique du fait de sa complexité. Le retour, initié dans le groupe 5 , sur l'organigramme élaboré à l'IUFM, aide les auteurs à le réajuster pour opérer une synthèse de l'atelier : axé sur les fonctions du professeur, il reflète, au delà de la diversité, l'unité de fonction des formateurs (fig. 7).

Figure 7. Organigramme de synthèse provisoire élaboré à la suite de l'atelier.

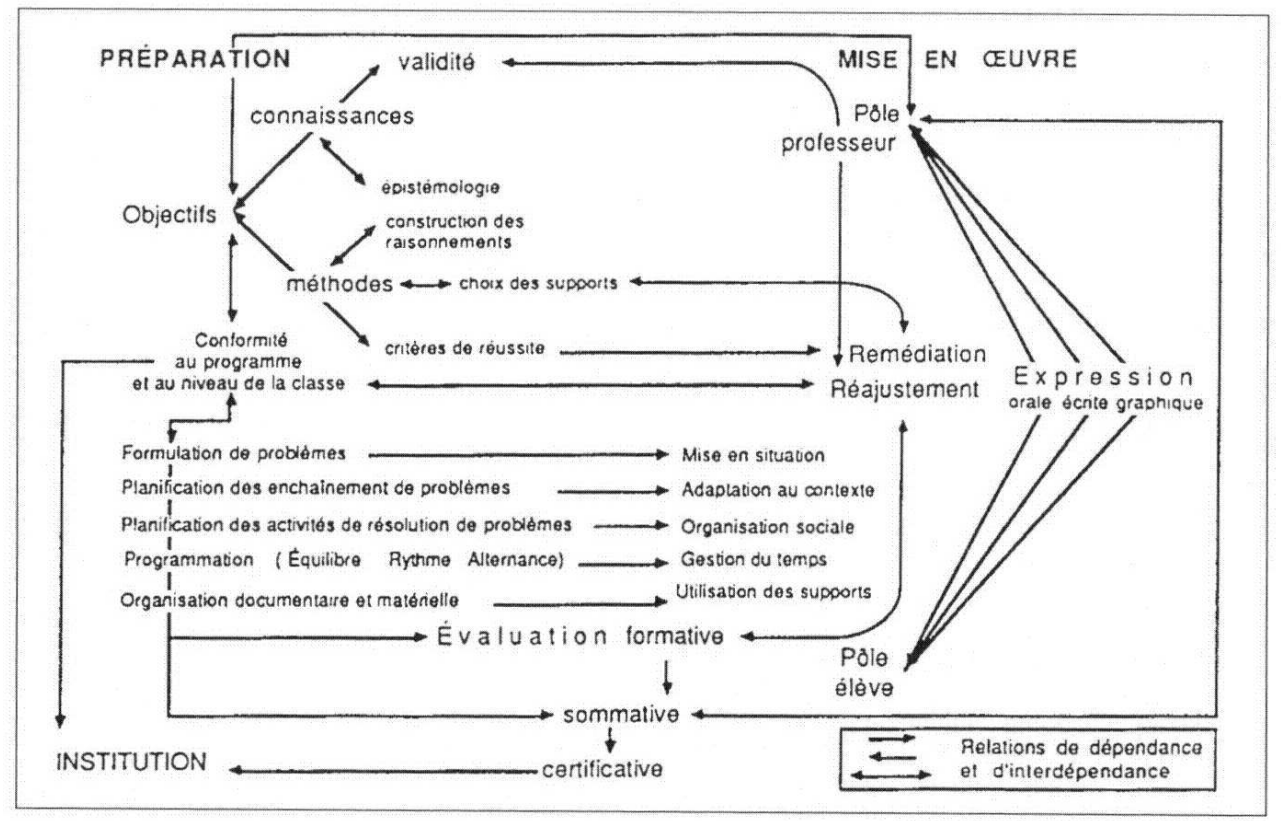

31 Pour le second point, les membres de l'atelier placent en priorité la validité des connaissances biologiques et la construction du savoir. C'est du côté des méthodes de validation-réfutation et des objets complexes que sont les organismes vivants qu'ils recherchent la spécificité de l'enseignement de la biologie, c'est-à-dire de la mise en scène des savoirs sur la vie et le vivant. La propulsion en avant, unanime, de la rubrique « formulation des problèmes » s'explique par son rôle constructeur du savoir biologique. C'est sans doute un acquis de la didactique des sciences qui n'existe pas ailleurs de façon aussi nette. Les situations pédagogiques proposées lors de l'énoncé de problèmes biologiques de l'enseignement ont pour caractéristique de s'appliquer à des objets liés à l'homme, à l'enfant, et de poser des questions touchant aux finalités éducatives, à l'éthique... L'enseignement de la géologie utilise aussi de telles situations de formulation préalable à la démarche scientifique, mais ses objets dépendent de variables moins nombreuses, à la fois moins accessibles et plus faciles à objectiver, à isoler dans une démarche modélisante. Par analogie, la situation d'observation de la classe, se rapproche 
de celle du vivant par la complexité des faits qui se résout par problèmes. L'organigramme a valeur de modèle élaboré à l'aide du formateur qui fournit matériaux et consignes, pour mettre les stagiaires en situation de recherche, comme ceux-ci le font eux-mêmes pour leurs élèves en biologie. Dans les deux cas, le codage des flèches s'impose pour une communication efficace de la réflexion.

\subsubsection{Perspectives d'utilisation de l'organigramme} proposer la construction d'organigrammes pour aider tuteurs et stagiaires enseignant la biologie à mettre au point et à s'approprier des grilles modulables, personnalisées, avec un fond commun. Elle motive des activités de formation (réalisation de trames conceptuelles, analyse de textes en histoire des sciences et réflexion sur quelques grands concepts biologiques, analyse de situations problèmes vécues par les stagiaires pour identifier les modes de pensée mis en œuvre...). Par la mise en évidence des nombreuses variables didactiques, elle facilite l'intégration par les stagiaires de la complexité de l'acte d'enseigner. Elle aide à prendre conscience des difficultés de l'observation: clans une situation, la diversité des plans et des sous-jacents théoriques à la valorisation de contenus ou de conceptions fait ressortir l'implication de l'observateur dans le choix des indices. Conscients des interactions entre les critères de l'outil, les enseignants sont conduits à mettre l'accent sur les relations et donc les réajustements. Mis en situation de préparer des séquences ou des progressions, ils prennent conscience de la nécessaire rencontre entre l'organisation des concepts transposés et les capacités des élèves : une double approche qui passe par une interaction entre l'élève et l'objet biologique à travers la résolution de problèmes, donc les activités de l'élève.

mise à disposition de fiches de différentes rubriques, à différents niveaux de formulation, depuis des simples extraits du dictionnaire jusqu'à des citations de spécialistes en sciences de l'éducation, aide les stagiaires clans leur élaboration, sans anticiper sur les interrelations possibles dans et entre les différentes catégories. La structuration du savoir, par exemple, y est définie comme la construction d'un système formé d'éléments ordonnés, organisés, en interrelation et en interaction, susceptible de transformations, de régulation. Elle implique l'idée de construction, de cadre théorique, de modèle. Son évolution évoque les ruptures suivies de reconstructions, d'équilibre provisoire, d'homéostasie susceptibles d'être à nouveau rompus par la dynamique de l'introduction de nouveaux obstacles. Une spécificité de la biologie réside dans ce fait qu'elle peut servir de modèle de complexité des relations entre ses différents niveaux de structuration interdépendants.

Réponse à une question, construction individuelle, interaction objet-sujet, obstacle, équilibration... autant d'éléments qui amènent à concevoir l'enseignement scientifique. Un travail sur la manière de faire ou d'être auprès des élèves permet à chaque stagiaire d'identifier ses priorités, de les analyser, de les faire évoluer et de les adapter aux situations d'enseignement-apprentissage dans le champ des sciences. Proposer aux stagiaires des situations problèmes, dans le champ de leur pratique comme dans celui de la biologie, leur permet de chercher les notions sous-jacentes, de lister les difficultés rencontrées, de construire un réseau conceptuel et un ensemble d'activités susceptibles de résoudre, à leur niveau et celui des élèves, les différents problèmes, pédagogiques et biologiques. 

l'élaborent. Par l'enrichissement que nous y avons trouvé, il confirme l'hypothèse que sa (re)construction est un facteur d'évolution. Face à la difficulté d'observer la classe, a fortiori de former à l'observation et à l'auto-observation, l'outil vaut par son caractère plastique. Il a aidé à définir la spécificité de la biologie qui réside dans la complexité de structuration des niveaux interdépendants du vivant, où toute validité dans un cadre de référence peut se trouver réfutée dans un autre cadre. L'approche systémique montre que la biologie peut servir de modèle d'organisation pour aider à structurer les concepts didactiques sous-jacents à la pratique de la classe. Il est intéressant de remarquer que des modèles d'apprentissage trouvent leurs racines dans la biologie : le modèle allostérique de Giordan né de l'enzymologie, le modèle oscillant de Scheefer inspiré de l'immunologie, mais aussi les équilibres homéostatiques du milieu forestier ou biostasiques d'un environnement géologique dont les ruptures nous servent de modèle au même titre que celles constatées à l'intérieur du vivant. Le cadre de référence d'un concept, didactique ou scientifique, dont nous définissons les limites dans le cadre de lecture évolutif de chacun, est lui-même un modèle de structuration du savoir inspiré des niveaux d'organisation du vivant. Nous profitons aussi des enseignements de l'histoire de la biologie pour faire appel de façon conjointe aux modes d'expression oral, écrit et graphique, plutôt que d'encourager le seul texte pour dire les priorités. C'est l'approche épistémologique qui est la nôtre pour structurer les organigrammes favorables à l'établissement des interdépendances entre théorie et pratique.

\section{BIBLIOGRAPHIE}

ALTET M., La formation professionnelle des enseignants, Paris, PUF, 264 p., 1994. 
AMIGUES R., « La description des situations d'enseignement : une nécessité et un obstacle pour la formation des maîtres ", in Cahiers de la recherche et du développement. Skholê, $\mathrm{N}^{\circ}$ 2, 1995, p. 217-230.

APPRENDRE A OBSERVER, Média Formation, DE, N 1, 1982, p. 116.

BERBAUM J. \& Coll. (HAGUENAUER C. et al), Guide pour l'observation de la classe : éléments pour une formation à l'observation des situations d'enseignement, Nancy, IREM, 69 p., 1976.

BERBAUM J., Étude systémique des actions de formation : introduction à une méthodologie de recherche, Paris, PUF, 239 p., 1982.

BRU M., Les variations didactiques dans l'organisation des conditions d'apprentissage, Toulouse, Éd. Universitaires, 165 p., 1991.

CLIGNET R., " Pour une analyse systématique des relations enseignant-enseigne ", in Revue française de pédagogie, $\mathrm{N}^{\circ} 43,1978$, p. 31-46.

HAGUENAUER C., « Le concept de cycle : un modèle et ses limites pour apprendre et pour évaluer l'abstraction ", in Actes de colloque, $5^{\mathrm{e}}$ rencontre : AEDB (Association Européenne de Didactique de la Biologie), Barcelone, 1993.

HAGUENAUER C., « Didactique et formation : formation disciplinaire et pratique pédagogique des professeurs de lycées et collèges en Biologie-Géologie ", in Pratiques et Formations, Nancy, CRDP, 1994, p. 47-78.

HAGUENAUER C., La flèche, outil transposable dans les science, Chamonix, A. Giordan, J.-L. Martinand \& D. Raichvarg Éditeurs, 1994, p. 261-266.

HAGUENAUER C., Les cycles, modèles réducteurs et/ou concept intégrateur du savoir scientifique en géologie, Ibid., 1995, p. 249-254.

HAGUENAUER C., (à paraître) Cadre de référence des concepts dans le cadre de lecture en évolution à partir de l'exemple des cycles en biologie, Les nouveaux modèles sur apprendre, Genève, Suisse, CBEIUBS, 1995.

POSTIC M. \& KETELE J.-M., Observer les situations éducatives, Paris, PUF, 1988.

\section{RÉSUMÉS}

L'observation de la pratique de professeurs confirmés ou des pairs novices est courante en formation et conduit à s'intéresser aux interrelations élèves -professeur - savoir pour démêler une situation pédagogique afin de donner du sens aux différentes actions. Plutôt que de fournir à l'observateur un questionnaire qui oriente sa recherche d'indicateurs, nous avons élaboré un organigramme, guide évoluable, favorisant la clarification conceptuelle de l'acte d'enseigner notamment la biologie.

Watching confirmed teachers or one's novice level peers exercising the teaching profession is common practice in the professional training of teachers and leads to an interest in the interrelationships between student, teacher and knowledge in order to untangle the pedagogical context and so as to draw out the meanings of the various actions. Rather than supplying the observer with a questionnaire, which would have directed his observations, we put together a flow chart to promote the conceptual clarification of the act of teaching, especially in biology. 
INDEX

Mots-clés : biologie, didactique, grille d'observation, style d'enseignement

Keywords : biology, classroom teaching style, didactic, observation criteria

\section{AUTEURS}

CHRISTIANE HAGUENAUER

IUFM de Lorraine, université de Nancy II

JEAN-PAUL DOSTE

IUFM de Lorraine, université de Nancy II 